\title{
Inhibiting tumor necrosis factor- $\alpha$ signaling attenuates postoperative cognitive dysfunction in aged rats
}

\author{
YUANZHONG MA ${ }^{1 *}$, QINGHAO CHENG ${ }^{2 *}$, ERMIN WANG $^{1}$, LEI LI $^{2}$ and XUBIN ZHANG ${ }^{3}$ \\ ${ }^{1}$ Department of Anesthesiology, Dezhou People's Hospital, Dezhou, Shandong 253045; \\ ${ }^{2}$ Department of Anesthesiology, China Meitan General Hospital, Beijing 100028; \\ ${ }^{3}$ Department of Orthopedics, Dezhou People's Hospital, Dezhou, Shandong 253045, P.R. China
}

Received July 16, 2014; Accepted March 26, 2015

DOI: $10.3892 / \mathrm{mmr} .2015 .3744$

\begin{abstract}
Postoperative cognitive dysfunction (POCD), commonly observed in elderly patients, is characterized by impaired concentration, memory and learning following surgery, and may persist for a long duration or progress into serious central nervous system diseases. It has been demonstrated neuroinflammation caused by surgery is involved in the development of POCD. However, the detailed molecular mechanism remains to be fully elucidated. The present study aimed to reveal the role of tumor necrosis factor (TNF)- $\alpha$ in the development of POCD in aged rats. Laparotomy was performed to mimic human abdominal surgery in the aged rats. Following surgery, the memory and learning functions were impaired, with significant upregulation of pro-inflammatory cytokines, including TNF- $\alpha$, interleukin (IL)- $1 \beta$, IL-4 and IL-6 in the hippocampal tissues. However, intracisternal administration of the TNF- $\alpha$ receptor antagonist, R-7050, during surgery attenuated these defects in cognitive function and inhibited the production of the pro-inflammatory cytokines in the hippocampal tissues. Furthermore, intracisternal administration of R-7050 inhibited the activation of the downstream nuclear factor- $\mathrm{\kappa B}$ and mitogen-activated protein kinase signaling pathway in the hippocampal tissues. Therefore, the results of the present study suggested a key role of the TNF- $\alpha$-mediated signaling pathway in the development of POCD.
\end{abstract}

Correspondence to: Dr Xubin Zhang, Department of Orthopedics, Dezhou People's Hospital, 1751 Xinhu Road, Dezhou, Shandong 253045, P.R. China

E-mail: doctorzhangxubin@163.com

*Contributed equally

Key words: postoperative cognitive dysfunction, tumor necrosis factor $\alpha$, neuroinflammation, aged

\section{Introduction}

Postoperative cognitive dysfunction (POCD) is characterized by impaired concentration, memory and learning following surgery. As POCD can persist for a prolonged period of time, it is detrimental to the health of millions of elderly patients worldwide, in addition to presenting a significant economic burden on society (1). As a result, the development of effective preventive or therapeutic targets for POCD is urgently required.

It has been suggested that neuroinflammation resulting from surgery is involved in the development of $\operatorname{POCD}(2,3)$. It is well-established that, shortly following surgery, the neuroinflammatory response-associated signaling pathways are activated, including nuclear factor (NF)- $\mathrm{BB}$. This can lead to the production and release of pro-inflammatory cytokines, including tumor necrosis factor (TNF)- $\alpha$, interleukin (IL)-1 $\beta$, IL-4, IL-6 and IL-8, and further neuroinflammatory responses in the brain (4-7). Therefore, effectively suppressing the activation of neuroinflammatory response-associated signaling pathways may offer potential for the prevention and treatment of POCD.

TNF- $\alpha$ is a multifunctional pro-inflammatory cytokine, predominantly secreted by macrophages (8). TNF- $\alpha$ is involved in the regulation of various biological processes, including cell proliferation, differentiation, apoptosis, lipid metabolism and coagulation, through binding to its receptors (9). In addition, TNF- $\alpha$ is able to activate neuroinflammatory-associated signaling pathways, including the NF- $\mathrm{kB}$ and mitogen-activated protein kinase (MAPK) signaling pathways $(4,5,7)$. Accordingly, TNF- $\alpha$ may be a potential preventive and therapeutic target in the treatment of POCD. However, the detailed role of TNF- $\alpha$ in surgery-induced POCD remains to be elucidated.

In the present study, in order to elucidate the role of TNF- $\alpha$ in surgery-induced POCD in aged patients, a rat model was used, in which laparotomy was performed to the aged rats in order to mimic human abdominal surgery. Subsequently, a Morris water maze (MWM) assay was performed to evaluate the cognitive functions of the rats following surgery, with or without the administration of the R-7050 TNF- $\alpha$ receptor antagonist. In addition, the expression levels of several key pro-inflammatory cytokines, and the activity of 
neuroinflammation-associated NF- $\mathrm{NB}$ signaling were examined in the hippocampal tissues of the aged rats.

\section{Materials and methods}

Animals and groups. The present study was approved by the Ethics Committee of Dezhou People's Hospital (Dezhou, China). Male Sprague-Dawley rats ( $\mathrm{n}=60 ; 24$ months old) were purchased from the Laboratory Animal Centre of Life Science Institute (Shanghai, China). The rats were housed separately under conditions of controlled temperature $\left(22 \pm 1^{\circ} \mathrm{C}\right)$ in a $12 \mathrm{~h}$ light/dark cycle, and were allowed free access to standard rat chow and sterile water. Each group contained 10 rats.

Surgery. Laparotomy was performed under anesthesia (Pelltobarbitalum Natricum; 5 mg/100 g; Sigma-Aldrich, Santa Clara, CA, USA). In the rats subjected to laparotomy, a $3 \mathrm{~cm}$ vertical incision was made at $\sim 0.5 \mathrm{~cm}$ below the lower right rib, and the incision penetrated the peritoneal cavity. The surgeon inserted an index finger into the opening and vigorously manipulated the viscera and musculature for $1 \mathrm{~min}$. Subsequently, sterile chromic gut sutures (Henan Songhe Medicines \& Health Products, Zhengzhou, China) were used to suture the peritoneal lining and muscle. In the sham-operated rats, the abdominal area was shaved and cleaned using $70 \%$ ethanol (Sigma-Aldrich) and the animals remained under anesthesia for the same duration as the rats in the laparotomy group

Intracisternal administration of the $R-7050 T N F-\alpha$ receptor antagonist. To investigate the role of TNF- $\alpha$ in the development of POCD following surgery in aged rats, a seperate group of the rats were administered with R-7050, a TNF- $\alpha$ receptor antagonist (EMD Biosciences, Inc., San Diego, CA, USA) during surgery under anesthesia. In brief, the dorsal aspect of the skull was shaved and cleaned using 70\% ethanol. A 27-gauge needle (Sigma-Aldrich), attached via PE50 tubing (Smiths Medical, Ashford, UK) to a $25 \mu$ l Hamilton syringe (Sigma-Aldrich), was inserted into the cisterna magna. To confirm entry into the cisterna magna, $2 \mu \mathrm{l}$ clear cerebral spinal fluid was drawn and released, following which $3 \mu$ l R-7050 was administered. In addition, a separate group of rats were shaved and cleaned, as above, and administered with $3 \mu \mathrm{l}$ sterile saline as a vehicle control.

MWM assay. All the rats were trained in the MWM five times each day for six consecutive days. Each rat was placed on a platform in the center of the MWM for $30 \mathrm{sec}$ and was then released into the water from an assigned release point. The rat was allowed to swim for $60 \mathrm{sec}$ to reach the platform. If unsuccessful, the rat was picked up and placed on the platform for another $30 \mathrm{sec}$. The swimming distance and the time taken to reach the platform were recorded using video tracking. The swimming distance and time taken to reach the platform were used to caculate the speed and then analyzed using MWM software (XR-XM101; Xinruan Information Technology, Shanghai, China). On postoperative days 1, 3 and 5, the MWM assay was repeated three times.

Western blotting. The proteins were extracted using a Nuclear and Cytoplasmic Protein Extraction kit (Thermo Fisher
Scientific, Waltham, MA, USA). The protein concentrations were determined using a Bradford DC Protein assay (Bio-Rad Laboratories, Inc., Hercules, CA, USA). Subsequently, the proteins were separated using 10\% SDS-PAGE (SigmaAldrich) and transferred onto a polyvinylidene difluoride (PVDF; Life Technologies, Carlsbad, CA, USA) membrane, which was then incubated with phosphate-buffered saline (PBS; Life Technologies) containing $50 \mathrm{~g} / 1$ skimmed milk at room temperature for $4 \mathrm{~h}$. Following this, the PVDF membrane was incubated with the following antibodies: Rabbit anti-TNF alpha (polyclonal; 1:100; cat. no. ab6671), rabbit anti-IL-1 $\beta$ (monoclonal; 1:50; cat. no. ab200478), rabbit anti-IL-4 (polyclonal; 1:50; cat. no. ab9622), rabbit anti-IL-6 (polyclonal; 1:50, cat. no. ab6672), mouse anti-c-JNK (monoclonal; 1:100; cat. no. ab46821), chicken anti-p-c-JNK (polyclonal; 1:100; cat. no. ab46821), rabbit anti-p38 (polyclonal; 1:50; cat. no. ab7952), rabbit anti-p-p38 (polyclonal; 1:50; cat. no. ab47363), rabbit anti-p65 (polyclonal; 1:50; ab16502) and mouse antiGAPDH (monoclonal; 1:100; cat. no. ab8245), respectively, at $37^{\circ} \mathrm{C}$ for $1 \mathrm{~h}$. Following washing with PBS three times, the PVDF membrane was incubated with HRP-conjugated goat anti mouse IgG (1:10,000; cat. no. ab186694), goat antirabbit $\operatorname{IgG}(1: 5,000$; cat. no. ab175773) or goat anti-chicken IgY (1:5,000; cat. no. ab175754). at room temperature for $1 \mathrm{~h}$. All antibodies were purchased from Abcam (Cambridge, UK). Chemiluminent detection was then performed using an Enhanced Chemiluminescence kit (Pierce Biotechnology, Inc., Rockford, IL, USA).

Statistical analysis. The data are expressed as the mean \pm standard deviation. Differences between two groups were determined using Student's t-test with SPSS software, version 17.0 (SPSS, Inc., Chicago, IL, USA). P<0.05 was considered to indicate a statistically significant difference.

\section{Results}

Aged rats exhibit defects in cognitive function following surgery. Prior to surgery, all the aged rats were trained in the MWM for 6 days. The swimming distances, time taken to reach the platform and speed in the MWM were used to evaluate the spatial memory function of the rats. As shown in Fig. 1, the swimming distance and time taken to reach the platform were significantly reduced during the six training days, indicating that their spatial memory gradually increased.

Subsequently, an MWM assay was performed on postoperative days 1,3 and 5. As shown in Fig. 2, the swimming distance and time taken to reach the platform were significantly increased on postoperative days 1 and 3, compared with the measurements obtained prior to surgery, suggesting that their spatial memory function was impaired shortly following surgery. However, on postoperative day 5 , the swimming distance and time taken to reach the platform were not significantly different, compared with those in the sham-operated control group, suggesting that their cognitive function had recovered.

Intracisternal administration of TNF- $\alpha$ receptor antagonist attenuates the defects in cognitive function in aged rats following surgery. To examine the role of TNF- $\alpha$ in POCD in aged rats, the rats in the present study received an intracisternal 

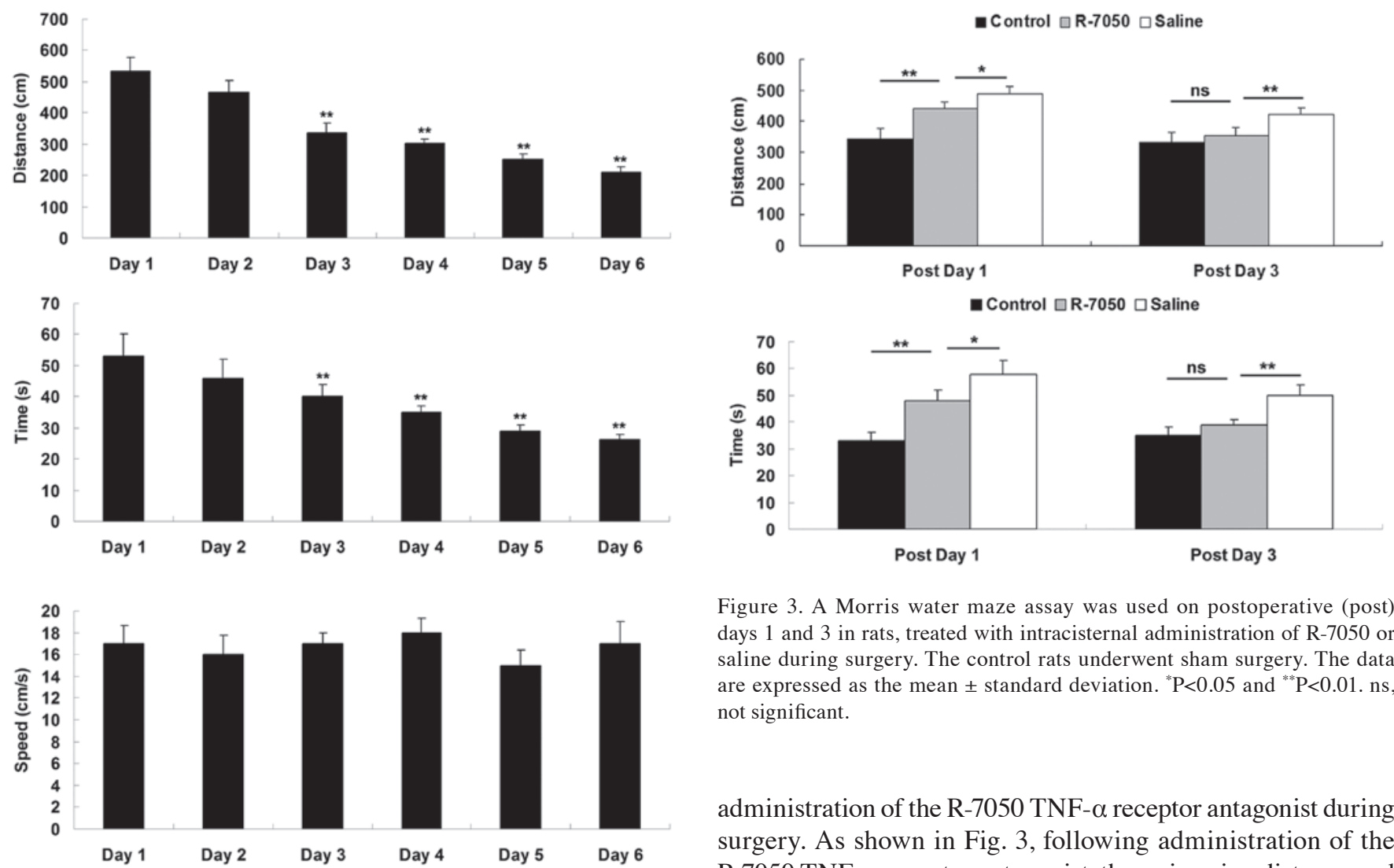

Figure 1. Prior to surgery, the aged rats were trained in the Morris water maze for 6 days. The swimming distance, time taken to reach the platform and the speed to the platform in the Morris water maze were calculated to evaluate the spatial memory function of the rats. The data are expressed as the mean \pm standard deviation. ${ }^{* *} \mathrm{P}<0.01$ vs. day 1 .

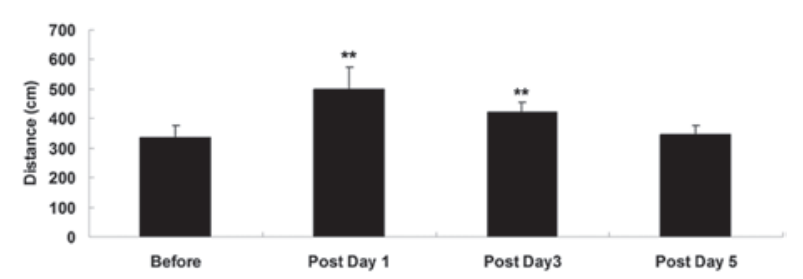

Figure 3. A Morris water maze assay was used on postoperative (post) days 1 and 3 in rats, treated with intracisternal administration of R-7050 or saline during surgery. The control rats underwent sham surgery. The data are expressed as the mean \pm standard deviation. ${ }^{*} \mathrm{P}<0.05$ and ${ }^{* *} \mathrm{P}<0.01$. ns, not significant.

administration of the R-7050 TNF- $\alpha$ receptor antagonist during surgery. As shown in Fig. 3, following administration of the R-7050 TNF- $\alpha$ receptor antagonist, the swimming distance and time taken for the aged rats to reach the platform were notably reduced on postoperative days 1 and 3, compared with the rats in the saline-treated group. However, the swimming distance and time taken to reach the platform in the aged rats treated with the R-7050 TNF- $\alpha$ receptor antagonist remained higher than those in the sham-operated control group on postoperative day 1 . These observations indicated that the intracisternal administration of the R-7050 TNF- $\alpha$ receptor antagonist notably attenuated the defects in spatial memory function observed in the aged rats shortly following surgery.

Intracisternal administration of the TNF- $\alpha$ receptor antagonist $R$-7050 inhibits the upregulation of pro-inflammatory cytokines in aged rats following surgery. The present study subsequently investigated the molecular mechanism underlying the effects of TNF- $\alpha$ further. Western blotting was used to determine the protein expression levels of pro-inflammatory cytokines, including TNF- $\alpha$, IL-1 $\beta$, IL-4 and IL-6, in the hippocampal tissues of aged rats on postoperative day 1 , with or without administration of the R-7050 TNF- $\alpha$ receptor antagonist. The rats in the shamoperated group were used as the controls. As shown in Fig. 4, the protein expression levels of TNF- $\alpha$, IL- $1 \beta$, IL-4 and IL-6 were significantly upregulated on postoperative day 1 , compared with the sham-operated group. However, the intracisternal administration of the TNF- $\alpha$ receptor antagonist significantly attenuated the surgery-induced upregulation of these pro-inflammatory cytokines. These data suggested that inhibiting TNF- $\alpha$-mediated pro-inflammatory signaling may effectively inhibit surgery-induced neuroinflammatory responses in aged rats.

Figure 2. Assessment using a Morris water maze test was performed prior to surgery (before) and on postoperative (post) days 1,3 and 5. The swimming distance, time taken to reach the platform and speed in the Morris water maze were calculated to evaluate the spatial memory function of the rats. The data are expressed as the mean \pm standard deviation ${ }^{* *} \mathrm{P}<0.01$ vs. before. 


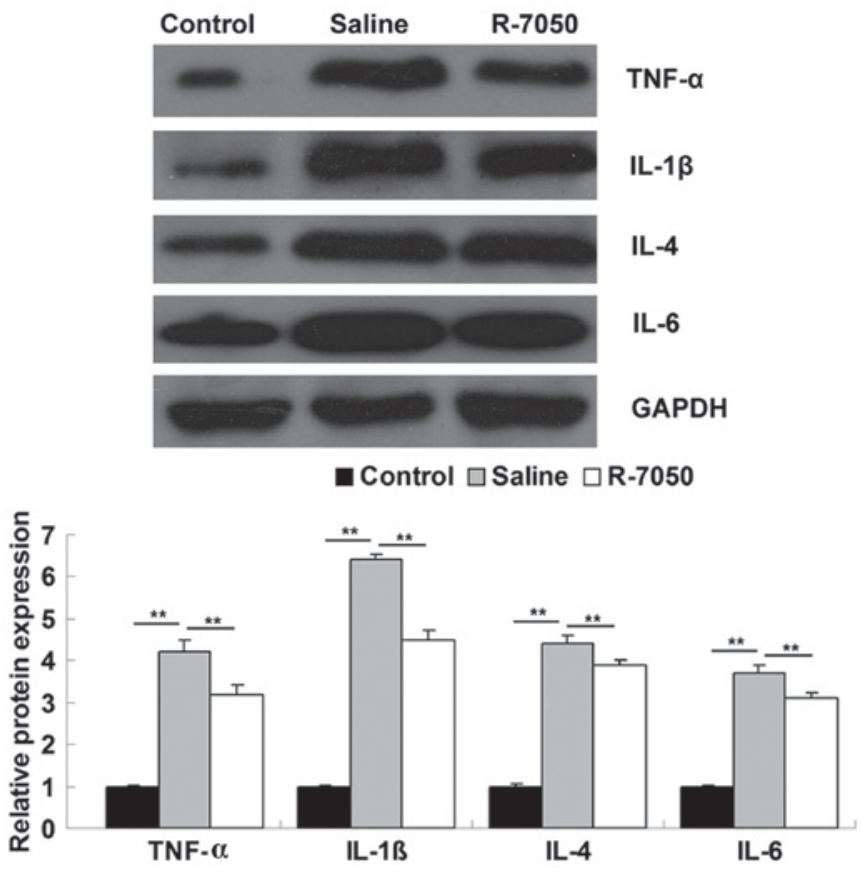

Figure 4. Western blotting was performed to determine the protein expression levels in the hippocampal tissues of aged rats on postoperative day 1 , following intracisternal administration of R-7050 or saline. The control rats underwent sham surgery. The data are expressed as the mean \pm standard deviation.** $\mathrm{P}<0.01$. TNF, tumor necrosis factor; IL, interleukin.

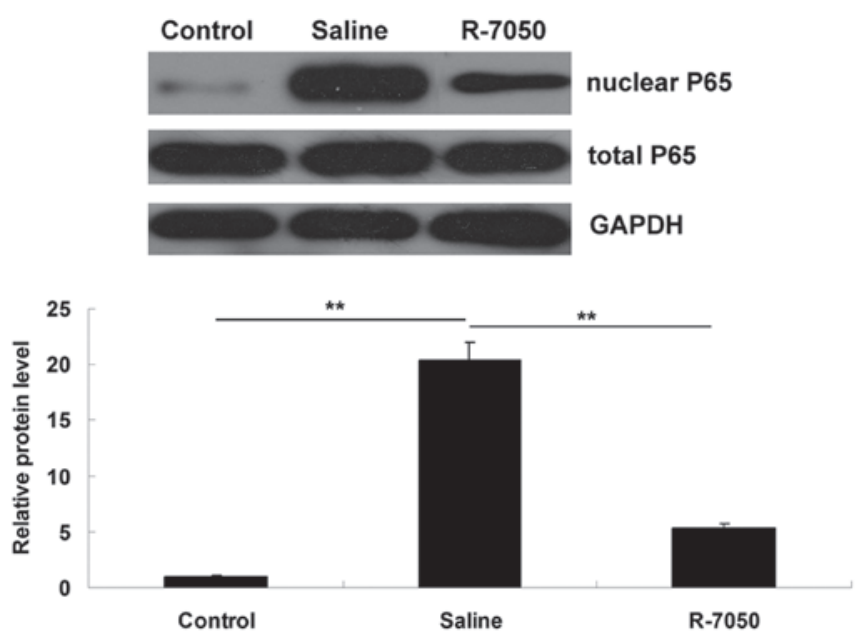

Figure 5. Western blotting was performed to determine the protein levels of nuclear P65 and total P65 in the hippocampal tissues of aged rats on postoperative day 1 following intracisternal administration of R-7050 or saline. The control rats underwent sham surgery. The data are expressed as the mean \pm standard deviation. ${ }^{* *} \mathrm{P}<0.01$.

pathway in aged rats following surgery. As NF- $\mathrm{B}$ is a downstream signaling factor of TNF- $\alpha$ (10), western blotting was performed to determine the activity of $\mathrm{NF}-\kappa \mathrm{B}$ signaling in the hippocampal tissues of the aged rats in each group. As shown in Fig. 5, compared with the control group, the protein levels of NF- $\mathrm{B}$ P65 in the nucleus were significantly upregulated on postoperative day 1 , and this upregulation was inhibited following intracisternal administration of the R-7050 TNF- $\alpha$ receptor antagonist. These observations suggested that the R-7050 TNF- $\alpha$ receptor antagonist effectively inhibited the
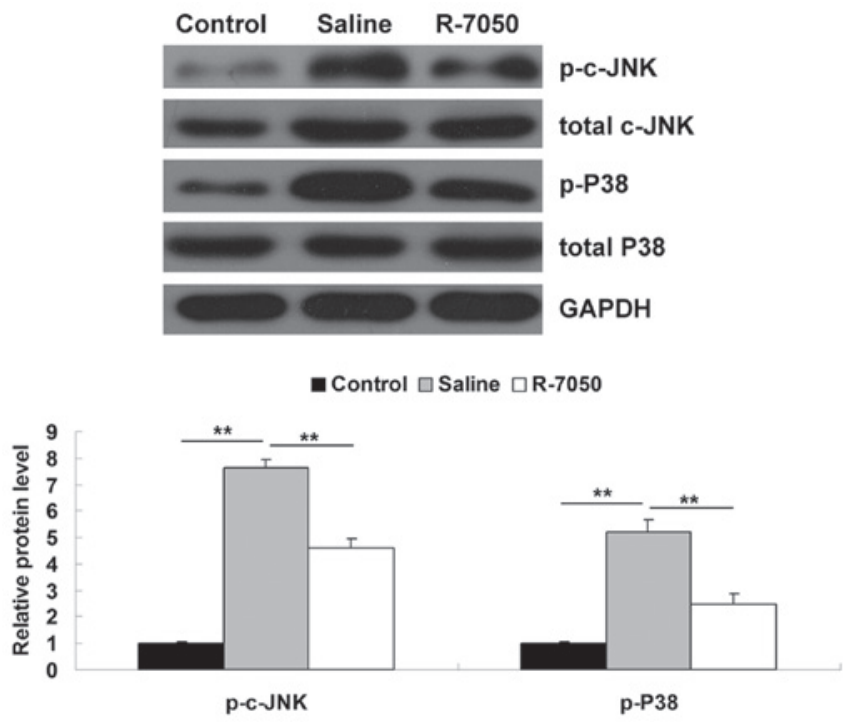

Figure 6. Western blotting was performed to determine the protein levels of p-c-JNK, p-p38 MAPK, total c-JNK and total p38 MAPK in the hippocampal tissue of aged rats on postoperative day 1 following intracisternal administration of R-7050 or saline. The control rats underwent sham surgery. The data are expressed as the mean \pm standard deviation. ${ }^{* *} \mathrm{P}<0.01$. c-JNK, c-Jun N-terminal kinases; MAPK, mitogen-activated protein kinase; $\mathrm{p}$-, phosphorylated.

surgery-induced activation of NF- $\mathrm{NB}$ signaling in the hippocampal tissues of the aged rats.

Intracisternal administration of the TNF- $\alpha$ receptor antagonist $R$-7050 suppresses the activation of the MAPK signaling pathway in aged rats following surgery. As MAPK signaling is also involved in TNF- $\alpha$-mediated inflammatory responses (11), western blotting was performed to examine the activity of c-Jun N-terminal kinases (c-JNK) and p38 MAPK signaling in the hippocampal tissues of the aged rats in each group. As shown in Fig. 6, the protein levels of phosphorylated c-JNK and phosphorylated p38 MAPK were significantly upregulated on postoperative day 1, compared with the levels in the control group. However, following intracisternal administration of the R-7050 TNF- $\alpha$ receptor antagonist, notable attenuation of the upregulation of these proteins was observed. Therefore, these data indicated that the inhibition of TNF- $\alpha$ effectively inhibited the surgery-induced activation of MAPK signaling in the hippocampal tissues of the aged rats.

\section{Discussion}

There is accumulating evidence to suggest that neuroinflammation may be involved in the development of POCD, as it is involved in cognitive defects in diseases of the central nervous system $(12,13)$. However, the detailed molecular mechanism remains to be elucidated. With the exception of advanced age as the most significant risk factor, surgical trauma is another risk factor for the development of POCD (1,14-16). In the present study, laparotomy was performed to mimic human abdominal surgery in aged rats. The data obtained demonstrated that that the laparotomy resulted in impaired cognitive function, while inhibiting TNF- $\alpha$, using the R-7050 TNF- $\alpha$ receptor antagonist significantly attenuated the laparotomy-induced defects 
in the cognitive functions of the aged rats. Inhibiting TNF- $\alpha$ inhibited the activation of $\mathrm{NF}-\kappa \mathrm{B}$ signaling and stimulated the production of key pro-inflammatory cytokines, including TNF- $\alpha$, IL-1 $\beta$, IL-4 and IL- 6 in the hippocampal tissues of the aged rats.

Previous studies have demonstrated that the development of POCD is associated with non-infectious neuroinflammatory responses $(3,17)$. As peripheral surgery has been observed to induce neuroinflammatory responses $(16,18)$, laparotomy was performed in the present study to mimic human abdominal surgery in aged rats. Peripheral surgery, including laparotomy cause sensitization, leading to enhanced neuroinflammation (19) and the release of peripheral pro-inflammatory cytokines, including TNF- $\alpha$, IL-1 $\beta$, IL-4 and IL-6 (20). These pro-inflammatory cytokines are able to enter the brain and further activate microglial cells, which causes further neuroinflammatory responses and brain injury, as described by Kannan et al (21). In the present study, the findings demonstrated that laparotomy resulted in a significant upregulation of pro-inflammatory cytokines in the hippocampal tissues of the aged rats. These observations were consistent with those of previous studies. Barrientos et al (22) also observed that splenectomy led to hippocampal-dependent memory impairment and upregulation in the levels of pro-inflammatory cytokines in the hippocampal tissues of aged rats on postoperative days 1 and 4 .

TNF- $\alpha$ has been observed to act on several signaling pathways through binding to its two receptors, TNF receptor 1 (TNFR1) and TNFR2. TNF-mediated signaling pathways are involved in $\mathrm{NF}-\kappa \mathrm{B}$ - and MAPK-mediated activation of inflammation $(10,11)$. Binding of TNF- $\alpha$ to TNFR 1 or TNF receptor-associated factor 2 can lead to activation of $\mathrm{NF}-\kappa \mathrm{B}$ signaling, which subsequently leads to production of pro-inflammatory cytokines, including TNF- $\alpha$, IL-1 $\beta$, IL-4 and IL-6 $(10,23,24)$. In the present study, it was demonstrated that intracisternal administration of a $\mathrm{TNF}-\alpha$ receptor antagonist suppressed the activation of the NF- $\kappa \mathrm{B}$ signaling pathway, with a resulting decrease in the production of TNF- $\alpha$, IL-1 $\beta$, IL- 4 and IL- 6 in the hippocampal tissues of the aged rats following surgery. These observations suggested that inhibiting TNF- $\alpha$ inhibited the surgery-induced neuroinflammation in the brain through downregulation of the $\mathrm{NF}-\kappa \mathrm{B}$ signaling-induced release of pro-inflammatory cytokines, and therefore, attenuated the defects in cognitive function in the aged rats.

MAPK signaling is also involved in TNF- $\alpha$-mediated inflammatory responses (11). c-JNK is a member of the MAPK family (25), and activation of TNF- $\alpha$-mediated signaling leads to transcriptional regulation, including c-JNK phosphorylation, to stimulate transcriptional activation by activator protein 1 (AP-1) (26). In addition, activation of the p38 MAPK signaling pathway also contributes to AP-1 activation, leading to the transcriptional activation of several genes involved in inflammation, including TNF- $\alpha$, IL-1 $\beta$, IL-4 and IL-6 (27-29). Accordingly, the activities of the MAPK signaling pathways, including c-JNK and p38 MAPK, were investigated in the present study following the inhibition of TNF- $\alpha$ in the hippocampal tissue of aged rats following peripheral surgery. The results of this investigation suggested that inhibiting TNF- $\alpha$ inhibited the surgery-induced activation of the c-JNK and p38
MAPK signaling pathway-induced release of TNF- $\alpha$, IL-1 $\beta$, IL-4 and IL-6 in the hippocampal tissues of the aged rats.

In conclusion, the results of the present study suggested that, in aged rats, upregulated TNF- $\alpha$ resulting from peripheral surgery, further activated the downstream NF- $\kappa \mathrm{B}$ signaling pathway, leading to increased release of pro-inflammatory cytokines, further extensive neuroinflammatory responses and, ultimately, to defects in cognitive function. Accordingly, targeting TNF- $\alpha$ may be a promising strategy for the prevention and treatment of POCD.

\section{References}

1. Colenkova AV, Bondarenko AA, Yu Lubnin A and Dzyubanova NA: Postoperative cognitive dysfunction in elderly patients. Anesteziol Reanimatol 4: 13-19, 2012 (In Russian).

2. Li M, Yong-Zhe L, Ya-Qun M, Sheng-Suo Z, Li-Tao Z and Ning-Ling P: Ulinastatin alleviates neuroinflammation but fails to improve cognitive function in aged rats following partial hepatectomy. Neurochem Res 38: 1070-1077, 2013.

3. Cao XZ, Ma H, Wang JK, et al: Postoperative cognitive deficits and neuroinflammation in the hippocampus triggered by surgical trauma are exacerbated in aged rats. Prog Neuropsychopharmacol Biol Psychiatry 34: 1426-1432, 2010.

4. Chen J, Buchanan JB, Sparkman NL, Godbout JP, Freund GG and Johnson RW: Neuroinflammation and disruption in working memory in aged mice after acute stimulation of the peripheral innate immune system. Brain Behav Immun 22: 301-311, 2008.

5. Garcia GE, Xia Y, Chen S, et al: NF-kappaB-dependent fractalkine induction in rat aortic endothelial cells stimulated by IL-1beta, TNF-alpha and LPS. J Leukoc Biol 67: 577-584, 2000.

6. Rosczyk HA,Sparkman NL and Johnson RW: Neuroinflammation and cognitive function in aged mice following minor surgery. Exp Gerontol 43: 840-846, 2008.

7. Terrando N, Monaco C, Ma D, Foxwell BM, Feldmann M and Maze M: Tumor necrosis factor-alpha triggers a cytokine cascade yielding postoperative cognitive decline. Proc Natl Acad Sci USA 107: 20518-20522, 2010.

8. Riches DW, Chan ED and Winston BW: TNF-alpha-induced regulation and signalling in macrophages. Immunobiology 195: 477-490, 1996.

9. Yang HL, Chang HC, Lin SW, et al: Antrodia salmonea inhibits TNF- $\alpha$-induced angiogenesis and atherogenesis in human endothelial cells through the down-regulation of NF- $\mathrm{BB}$ and up-regulation of Nrf2 signaling pathways. J Ethnopharmacol 151: 394-406, 2014.

10. Song HY, Regnier CH, Kirschning CJ, Goeddel DV and Rothe M: Tumor necrosis factor (TNF)-mediated kinase cascades: Bifurcation of nuclear factor-kappaB and c-jun N-terminal kinase (JNK/SAPK) pathways at TNF receptor-associated factor 2. Proc Natl Acad Sci USA 94: 9792-9796, 1997.

11. Yuasa T, Ohno S, Kehrl JH and Kyriakis JM: Tumor necrosis factor signaling to stress-activated protein kinase (SAPK)/Jun NH2-terminal kinase (JNK) and p38. Germinal center kinase couples TRAF2 to mitogen-activated protein kinase/ERK kinase kinase 1 and SAPK while receptor interacting protein associates with a mitogen-activated protein kinase kinase kinase upstream of MKK6 and p38. J Biol Chem 273: 22681-22692, 1998.

12. Hu Z, Ou Y, Duan K and Jiang X: Inflammation: A bridge between postoperative cognitive dysfunction and Alzheimer's disease. Med Hypotheses 74: 722-724, 2010.

13. Yu D, Corbett B, Yan Y, et al: Early cerebrovascular inflammation in a transgenic mouse model of Alzheimer's disease. Neurobiol Aging 33: 2942-2947, 2012.

14. Abildstrom H, Rasmussen LS, Rentowl P, et al: Cognitive dysfunction 1-2 years after non-cardiac surgery in the elderly. ISPOCD group. International study of post-operative cognitive dysfunction. Acta Anaesthesiol Scand 44: 1246-1251, 2000.

15. Boodhwani M, Rubens FD, Wozny D, et al: Predictors of early neurocognitive deficits in low-risk patients undergoing on-pump coronary artery bypass surgery. Circulation 114 (Suppl 1): I461-I466, 2006.

16. Canet J, Raeder J, Rasmussen LS, et al: Cognitive dysfunction after minor surgery in the elderly. Acta Anaesthesiol Scand 47: 1204-1210, 2003. 
17. Deiner S and Silverstein JH: Postoperative delirium and cognitive dysfunction. Br J Anaesth 103 (Suppl 1): i41-i46, 2009.

18. Kamer AR, Galoyan SM, Haile M, et al: Meloxicam improves object recognition memory and modulates glial activation after splenectomy in mice. Eur J Anaesthesiol 29: 332-337, 2012.

19. Hains LE, Loram LC, Taylor FR, et al: Prior laparotomy or corticosterone potentiates lipopolysaccharide-induced fever and sickness behaviors. J Neuroimmunol 239: 53-60, 2011.

20. Didem B, Huseyin B, Osman Y, Yasemin B, Necati G and Canan T: Early effects of laparotomy and laparoscopy on bacterial behavior and proinflammatory cytokines on bacterial peritonitis in rats I: Escherichia coli. J Pediatr Surg 43: 1494-1501, 2008.

21. Kannan S, Saadani-MakkiF, Muzik O, et al: Microglial activation in perinatal rabbit brain induced by intrauterine inflammation: Detection with 11C-(R)-PK11195 and small-animal PET. J Nucl Med 48: 946-954, 2007.

22. Barrientos RM, Hein AM, Frank MG, Watkins LR and Maier SF: Intracisternal interleukin-1 receptor antagonist prevents postoperative cognitive decline and neuroinflammatory response in aged rats. J Neurosci 32: 14641-14648, 2012.

23. Hoesel B and Schmid JA: The complexity of NF- $\kappa \mathrm{B}$ signaling in inflammation and cancer. Mol Cancer 12: 86, 2013.

24. Muñoz A and Costa M: Nutritionally mediated oxidative stress and inflammation. Oxid Med Cell Longev 2013: 610950, 2013.
25. Arslan F, Lai RC, Smeets MB, et al: Mesenchymal stem cell-derived exosomes increase ATP levels, decrease oxidative stress and activate PI3K/Akt pathway to enhance myocardial viability and prevent adverse remodeling after myocardial ischemia/reperfusion injury. Stem Cell Res 10: 301-312, 2013.

26. Chen WL, Sheu JR, Hsiao CJ, Hsiao SH, Chung CL and Hsiao G: Histone deacetylase inhibitor impairs plasminogen activator inhibitor-1 expression via inhibiting TNF- $\alpha$-activated MAPK/AP-1 signaling cascade. Biomed Res Int 2014: 231012, 2014.

27. Lee IT, Lin CC, Cheng SE, Hsiao LD, Hsiao YC and Yang CM: TNF-alpha induces cytosolic phospholipase A2 expression in human lung epithelial cells via JNK1/2- and p38 MAPK-dependent AP-1 activation. PLoS One 8: e72783, 2013.

28. Qiu Q, Xiong W, Yang C, et al: Lymphocyte-derived microparticles induce apoptosis of airway epithelial cells through activation of p38 MAPK and production of arachidonic acid. Apoptosis 19: 1113-1127, 2014

29. Ma JQ, Ding J, Zhang L and Liu CM: Ursolic acid protects mouse liver against $\mathrm{CCl} 4$-induced oxidative stress and inflammation by the MAPK/NF- $\kappa$ B pathway. Environ Toxicol Pharmacol 37: 975-983, 2014. 